\title{
Non Hodgkin Lymphomas (NHL) in the Pediatric Oncology Unit of the Gabriel Touré Teaching Hospital, Bamako Mali
}

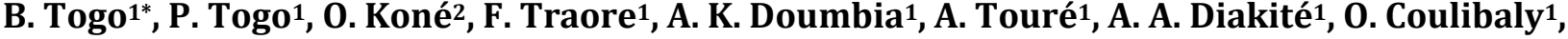

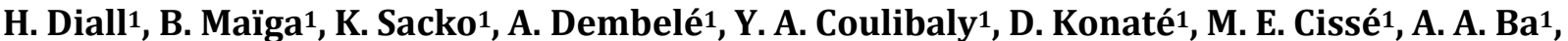 \\ F. L. Diakité1, L. N. Sidibé1, A. Doumbia1 ${ }^{1}$ H. Konaré1, L. B. Maïga1 ${ }^{1}$ C. B. Traoré ${ }^{3}$
}

\author{
${ }^{1}$ Department of Pediatrics, Gabriel Touré Teaching Hospital, Bamako, Mali \\ ${ }^{2}$ National Institute of Research in Public health, Bamako, Mali \\ ${ }^{3}$ Pathology Service of Point G Teaching Hospital, Bamako, Mali \\ Email: *togoboubacar2000@yahoo.fr
}

How to cite this paper: Togo, B., Togo, P., Koné, O., Traore, F., Doumbia, A.K., Touré, A., Diakité, A.A., Coulibaly, O., Diall, H., Maïga, B., Sacko, K., Dembelé, A., Coulibaly, Y.A., Konaté, D., Cissé, M.E., Ba, A.A., Diakité, F.L., Sidibé, L.N., Doumbia, A., Konaré, H., Maïga, L.B. and Traoré, C.B. (2019) Non Hodgkin Lymphomas (NHL) in the Pediatric Oncology Unit of the Gabriel Touré Teaching Hospital, Bamako Mali. Open Journal of Pediatrics, 9 , 309-316.

https://doi.org/10.4236/ojped.2019.94030

Received: October 9, 2019

Accepted: December 2, 2019

Published: December 5, 2019

Copyright $\odot 2019$ by author(s) and Scientific Research Publishing Inc. This work is licensed under the Creative Commons Attribution International License (CC BY 4.0).

http://creativecommons.org/licenses/by/4.0/ (c) (i) Open Access

\begin{abstract}
Background: Non-Hodgkin lymphomas are the first childhood cancer in sub-Saharan Africa. Objective: The purpose of this study was to assess non-Hodgkin lymphomas cases in our setting. Methodology: A retrospective and descriptive study carried out in the pediatric oncology unit of the Gabriel Touré Teaching Hospital Bamako over 10 years from 1st January 2005 to 31th December 2015. Results: We exploited 274 (21.6\%) cases of Non-Hodgkin Lymphoma out of 1295 cancer cases registered, the age group 6 - 10 years was the most represented (46.4\%); the male sex was predominant with a sex-ratio of 1.8; digestive signs were the most common signs of discovery (44.2\%) followed by maxillary swelling (42.7\%); the majority of patients $(52.9 \%)$ consulted between 1 and 3 months after the onset of signs; the malnutrition rate was $39.8 \%$, of which $24.1 \%$ were severe cases and $15.7 \%$ were moderate rate. Abdominal localization was the most common (43.1\%) followed by maxillofacial localization (33.9\%). Almost all were Burkitt type cytology (92.7\%), the majority (73.4\%) were in Murphy stage III. Almost all (96\%) had received chemotherapy and the modified LMB 01 protocol was widely used (62.4\%). The majority of patients (85\%) were chemosensitive at day 7 or after the third cyclophosphamide injection but at the end of induction only $31 \%$ were in complete remission. Gastrointestinal toxicity was the most common (37.13\%) followed by hematologic toxicity $35.09 \%$; $9.12 \%$ of patients were lost of follow-up and $22.26 \%$ died. Tumor progression was the most common cause of death $(60.66 \%)$ followed by infection $(21.31 \%)$. Conclusion: In light of these
\end{abstract}


findings, the late diagnosis and the poor management of NHL, as well as the limited ability to primarily treat metabolic complications, explain the high case-fatality rate, hence the important role of early diagnosis and treatment multidisciplinary.

\section{Keywords}

Childhood Lymphomas, Gabriel TOURE Teaching Hospital, Mali

\section{Introduction}

The global cancer burden is set to increase to 21.6 million cases by 2030 mainly in Latin America, Asia and Africa [1].

Over $60 \%$ of the total number of cancer cases in the world occur in Africa, central Asia and South America. These regions register about $70 \%$ of cancer deaths across the world, a situation compounded by the absence of early screening and access to treatment [2].

Even though cure is possible in most childhood cancer cases, the death rate in the world remains high given that $80 \%$ of children affected by cancer live in developing countries where no effective treatment is available. As a result, we note a survival rate of about $20 \%$ in these countries [3].

The Non-Hodgkin lymphomas fall into a heterogeneous group of malignant proliferations of the lymphoid tissue. The major types identified in childhood include: the Burkitt lymphoma (BL), the lymphoblastic lymphoma (LL), the diffuse large B cell lymphoma and the anaplastic lymphoma. The burden of NHL varies according to countries. It is estimated to range between 3.5 and 11.5 new cases for 100,000 inhabitants a year [4].

In Mali, NHLs were the leading causes of childhood cancers between 2005 and 2010 as registered at Gabriel Touré Teaching Hospital of Bamako with 33\% of cases [5]. Several studies were conducted on Burkitt lymphoma cases in Mali however very few studies were conducted on other types of Non-Hodgkin lymphomas, hence the relevance of our study in the pediatric oncology unit of Gabriel Touré Teaching Hospital in Bamako with the aim of describing various aspects of the NHLs admitted in our unit.

\section{Materials and Method}

\subsection{Setting of the Study}

This study was conducted in the pediatric oncology unit of the pediatric department of Gabriel Touré Teaching Hospital with ten beds, a staff of 4 pediatric oncologists and 4 nurses.

\subsection{Study Period and Type}

This was a retrospective and descriptive study based on patient files treated from 
1st January 2005 to 31 December 2015.

\subsection{Inclusion Criteria}

All children aged from 0 to 15 years with a cytologically and histologically confirmed NHL diagnostic.

\subsection{Diagnostic and Treatment}

NHL's diagnosis was established by cytology and pathology. The cytology blades were read by two pediatric oncologists then confirmed by the hematology laboratory of Robert Débré Hospital in Paris.

With respect to other types of lymphoma, biopsies were sent to the pathology laboratory of the University of Munster in Germany. The search for blasts in the cerebrospinal fluid was not done and screening for neuro-meningeal involvement was based on clinical assessment.

More sophisticated tests such as molecular biology, cytogenetic and immunohistochemistry could not be carried out. Blood electrolytes were carried out only with a few patients who had the financial resources to pay for them. Blood products and antibiotics were available for supportive care. Chemotherapy protocols used were from The French-African Pediatric Oncology Group (FAPOG) who provided the drugs.

Prior to any chemotherapy, a preliminary treatment were carried out with each patient; it involved deworming, treatment of any possible infection, refeeding.

The cyclo-Burkitt, a monotherapy with cyclophosphamide alone for localized forms of Burkitt lymphoma consisted of an intra venous injection of $1.2 \mathrm{~g} / \mathrm{m}^{2}$ of cyclophosphamide associated with an intraspinal injection of $15 \mathrm{mg}$ of methotrexate and $15 \mathrm{mg}$ of hydrocortisone. After 3 cyclo Burkitt and for stages 1 and 2, a complete remission would lead to a stoppage of any treatment and a surveillance is set up. A small residue was then treated through surgery removal. If there were to be a poor response to three cyclo Burkitt for stages I and II, a more aggressive treatment protocol of the LMB type (a French B malignant lymphoma treatment protocol) were set up. In case of a positive response in stage III was observed, three additional cyclo Burkitt were administered at 15-day intervals. A negative response would lead to a second line chemotherapy.

\subsection{Statistical Analysis}

The main variables, namely age, sex, socio economic parameters, clinical signs, location, evolutive stage, toxicity of drugs used, and outcome were recorded and analyzed on SPSS (Chicago, USA).

\subsection{Limitation of Our Study}

Due to extreme poverty of our patients' parents, some tests like immunophenotyping, cytogenetics, and molecular biology have not been done. 


\section{Results}

\subsection{Socio-Demographic Features}

We exploited 274 files of NHL out of 1295 of cancer cases registered; $21.16 \%$ of cases. The age group 6 - 10 years was the most represented (46.4\%). The sex ratio (M/F) was 1.8 . We have recorded $9.5 \%$ of patients from neighboring countries; $62 \%$ of fathers were illiterate, of whom $69.3 \%$ were farmers; $82.5 \%$ of mothers were illiterate of whom $96.7 \%$ were housewives.

\subsection{Clinical Features (Table 1 and Table 2)}

Digestive signs were the most frequent signs of discovery (46\%) followed by jaw swelling (42.7\%). Over half of the patients (53\%) had consulted within 1 to 3 months, only $6.9 \%$ had consulted within less than a month. The malnutrition rate stood at $39.8 \%$ ( $24.1 \%$ of severe cases and $15.7 \%$ of moderate cases). Abdominal location was the most represented (43.1\%) followed by location at the jaw (33.9\%). With $93.1 \%$ of patients the diagnostic was established through cytology (from the mass: $83.1 \%$; from lymph nodes: $7.7 \%$; from ascite fluid: $1.5 \%$ ). Burkitt lymphoma accounted for $92.7 \%$ of cases including $73.4 \%$ of cases at Murphy stratification stage II. Tumor extension involved lymph nodes in $40 \%$ of cases and bone marrow in $4.4 \%$ of cases. All patients were treated by chemotherapy. The steroid sensitivity at day 7 was $85 \%$ of patients.

Upon completion of the induction, $31 \%$ of patients reached complete remission and $39.1 \%$ partial remission. Three of our patients went through surgery. During treatment, complications due to toxicity of drugs happened in $62.8 \%$ of cases; $37.13 \%$ of which were digestive related. Evolution was known with $90.88 \%$ of patients and $54.74 \%$ were at complete remission; $22.26 \%$ deceased, while $13.86 \%$ abandoned treatment and $9.12 \%$ were lost of follow-up. Deaths were due to tumor progression in $60.66 \%$ of cases and to infection in $21.31 \%$ of cases.

Table 1. Different signs at diagnosis.

\begin{tabular}{ccc}
\hline Signs at diagnosis & Numbers & Percentage (\%) \\
\hline Abdominal pains & 38 & 13.9 \\
Abdominal distension & 23 & 8.4 \\
Abdominal mass & 60 & 21.9 \\
Jaw swelling & 117 & 42.7 \\
Peripheral adenopathies & 23 & 8.4 \\
Loossening of teeth & 1 & 0.4 \\
Transit disorder & 5 & 1.8 \\
Shortness of breath & 4 & 1.5 \\
vena cava superior syndrome & 1 & 0.4 \\
motor deficiency & 1 & 0.4 \\
Pleural effusion syndrome & 1 & 0.4 \\
Total & 274 & 100 \\
\hline
\end{tabular}


Table 2. Patients distribution based on location of lymphomas.

\begin{tabular}{ccc}
\hline Location & Number & Percentage (\%) \\
\hline Abdominal & 118 & 43.1 \\
Jaw & 93 & 33.9 \\
Abdominal + jaw & 25 & 9.1 \\
peripheral nodes & 12 & 4.4 \\
Abdominal + peripheral nodes & 10 & 3.6 \\
Multiples & 3 & 1.1 \\
Chest + peripheral nodes & 3 & 1.1 \\
Abdominal + thoracic & 3 & 1.1 \\
Chest & 3 & 1.1 \\
Bones and stomach & 2 & 0.7 \\
Ear nose throat (cavum, amygdales) & 2 & 0.7 \\
Total & 274 & 100 \\
\hline
\end{tabular}

\section{Discussion}

In our study the NHLs accounted for $21.16 \%$ of cancer cases making it the leading cause for hospitalization due to cancer in our pediatric oncology unit at Gabriel Touré Teaching Hospital.

In the West the NHLS account for $5 \%$ to $10 \%$ of all malign tumors of the child and is the third cause of cancer after brain tumors and leukemia. In Sub Saharan Africa they lead the statistics in all pediatric oncology units [6] [7] [8].

About 500 cases are diagnosed annually in the USA and 7 in one million cases in Europe making the NHLS the third major cause of consultation in these countries after leukemia and brain tumors [9] [10].

Most of the patients were from Koulikoro, the second region of Mali, and $24.5 \%$ of cases. This could be explained by the fact that the study was conducted in a Bamako center where some neighborhoods are part administrative districts of Koulikoro. We also recorded patients from neighboring countries accounting for $9 \%$ of cases. In some of those countries they lack a unit for the care to patients, however some patients came to our unit for its proximity.

Most of the fathers of these patients were farmers (69.3\%) and were not educated (62\%). These results are consistent with the findings of the 4th demographic and health survey of Mali (EDSM-IV) which show that $53 \%$ of men lived from agriculture, fishing and breeding of whom $64 \%$ had no education [11].

Almost all mothers were housewives (96.7\%), three fourths of them (82.5\%) were not educated. According to EDSM-IV, 73\% of women are not educated. The mother's education level has a positive impact on the socio-economic well being of the household as well as on the use of health services.

We noted the predominance of males (64.6\%) with a sex ratio of 1.8. This male predominance was noted in several studies in Africa involving NHLs of the child namely in Burkina Faso, Togo and Madagascar with ratios of 1.17; 1.5 and 
2.07 respectively [12] [13] [14].

Frequency also varies according to ethnic group, in the US between 1985 and 1990 and based on the epidemiology and final results program of the National Cancer Institute (NCI), the average annual burden of cancer in Caucasian children was 9.1 million and 4.1 million for blacks [15]. In equatorial Africa, $50 \%$ of cancers in children are represented by the Burkitt lymphoma whose burden is between 5 to 10 million children under 16 years [16]. Endemic regions include New Guinea, while North Africa and South America are regions with a medium level burden [17].

The age group 6 - 10 years was the most represented (46.4\%) with extreme age of 1 and 15 years and a mean age of 8 . These results are similar to those of the initial study conducted in our unit in 2008 where the most affected age group was the 6 - 10 years [18].

This same finding was reached by Adon, A., et al. in France who reported in their study that the mean age was 7 or 8 [19].

The NHLs can occur at any age but are quite rare before age 2 [20].

The time before consulting from $1-3$ months was found in 53\% of cases. In our countries where parents tend to first consult with traditional healers before visiting a health center, this tends to extend diagnostic deadline. This deadline of $1-3$ months was found in the study by Segnet in Togo [13].

In Europe and USA, 1/3 of pediatric lymphomas are represented by lymphoblastic lymphomas (LL), 1/2 are small cell lymphomas (Burkitt and Burkitt like) and the rest is dominated by large cell lymphomas while in some regions (Southern India) most of the NHLs are lymphoblastic related [21] [22].

Digestive signs (abdominal mass, pains abdominal extension) were signs that helped discover the disease in $46 \%$ of the cases. This was a result of the frequency of the abdominal involvement. whereas jaw location accounted for 36\% of cases, a contrary to study by A. Y. Segbena in Togo and F. Rafaramino in Madasgascar where facial and jaw locations are predominant [11] [12] [13].

Most Burkitt lymphomas were at the stage III of Murphy (73.4\%). Our proportion of the advanced stage was higher than the cases described in other studies [23]. As it was not possible to screen for blasts in the spinal fluid, nervous system extension was assessed only clinically which tends to minimize frequency of stage IV. We did not find any case of the stage I of Murphy. This high frequency of the advanced stages could be due to delay in diagnosis.

Most of our patient, $54.74 \%$ were in complete remission at the end of chemotherapy.

A FAPOG multicentric study on Burkitt lymphoma treatment based on cyclophosphamide monotherapy found that complete remission was at $47 \%$ with $33 \%$ of event free survival. In a previous study on maxillofacial Burkitt lymphoma carried out in our unit the rate of complete remission was $37.5 \%$ after 3 cyclophosphamide courses [19].

About $9 \%$ of cases were lost of follow up after chemotherapy and most often in complete remission. At the end of chemotherapy and in complete remission 
some parents living far from the city center do not deem it necessary to return to our center for follow up. We recorded a small number of patients dropping out of treatment (13.86\%) as the cures were performed in hospital and parents were impressed by rapid regression of abdominal mass.

The mortality rate was $22.26 \%$. This rate is comparable to that of A.Y. Segbena in Togo, F. Rafaramino in Madagascar and S. Ouedraogo in Burkina which were $25 \%, 22.5 \%$ and $18 \%$ respectively [12] [13] [14].

All NHL patients were treated according to protocols of the Burkitt type lymphoma regardless of the pathological features (lymphoblastic, large cell anaplastic, broad large B cell) because immune phenotyping was not available. This therapy was chosen based on the long duration of the $\mathrm{T}$ lymphoma protocols whose costs are too high for our resource poor countries [23]. In addition, we noted inadequate supportive care and unavailability of labile blood products.

\section{Conclusion}

Despite the limitation of our study, we learned that NHL are the leading cause of childhood cancer in our facility. Improvement of supportive care and early detection are needed to raise the overall survival.

\section{Conflicts of Interest}

The authors declare no conflicts of interest regarding the publication of this paper.

\section{References}

[1] Steward, B.N. and Wild, C.M. (2014) World Cancer Report 2014.

[2] Valsecchi, M.G. and Steliarova-Foucher, E. (2008) Cancer Registration in Developing Countries: Luxury or necessity. Lancet Oncology, 9, 159-167. https://doi.org/10.1016/S1470-2045(08)70028-7

[3] Lam, C.G., et al. (2019) Science and Health for All Children with Cancer. Science, 363, 1182-1186. https://doi.org/10.1126/science.aaw4892

[4] Brugières, L., Rodary, C. and Clavel, J. (2008) Epidémiologie des cancers. In: Kalifa, C. and Oberlin, O., Eds., Cancvers de Penfant, Flammarion, Paris, 3-13.

[5] Togo, B., Traore, F., Togo, A.P., Togo, P., Diakite, A.A., Traore, B., et al. (2014) Epidémiologie et pronostic des cancers pediatriques au CHU Gabriel-Toure de Bamako (Mali). Médecine et Santé Tropicales, 24, 68-72. https://doi.org/10.1684/mst.2014.0291

[6] West, R. (1984) Childhood Cancer Mortality: International Comparisons 1955-1974. World Health Statistics, 3, 798.

[7] Li, J., Thompson, T.D., Miller, J.W., Pollack, P.A. and Stewart, S.C. (2008) Cancer Incidence among Children and Adolescents in the United States, 2001-2003. Pedaitrics, 121, e1470. https://doi.org/10.1542/peds.2007-2964

[8] Traoré, F., Coze, C., Atteby, J.J., André, N., Moreira, C., Doumbe, P., et al. (2011) Cyclophosphamide Monotherapy in Children with Burkitt Lymphoma: A Study from the French-African Pediatric Oncology Group (GFAOP). Pediatric Blood \& Cancer, 56, 70-76. 
[9] Patte, C. (1998) Non-Hodgkinien Lymphoma. European Journal of Cancer, 34, 359-363. https://doi.org/10.1016/S0959-8049(97)10142-3

[10] Shad, A. and Magrath, I. (1997) Non-Hodgkin's Lymphoma. Pediatrics Clinics of North America, 44, 863-890. https://doi.org/10.1016/S0031-3955(05)70534-4

[11] République du Mali (2006) Enquête démographique et de Santé du Mali, $4^{\mathrm{e}}$ édition (EDSM-IV).

[12] Ouedraogo, S. (2013) Devenir des patients souffrant de lymphome de burkitt stades III ou IV pris en charge selon le protocole lmb09 dans le service de pédiatrie du CHU-YO du 15 mars 2009 au 14 mars 2012. Thèse médecine, Ouagadougou 2013.

[13] Segbena, A.Y., Kueviakue, M., Vovor, A., Tatagan-agbi, K., Assimadi, K., Napo-Koura, G.A. and Kpodzro, K. (1997) Le lymphome de Burkitt au Togo, aspects épidémiologique, clinique, thérapeutique et évolutif. Médecine d Afrique Noire, 44.

[14] Rafaramino, F., Maminirina, R.A.M., Razafindrabe, J.A.B., Rabarijaona, L., Randriamampandry, A. and Rakotobe, P. (2001) Le lymphome de Burkitt de l'enfant à Madagascar. Formes anatomo-cliniques, aspects thérapeutiques et évolutifs. Bulletin de la Société de Pathologie Exotique, 94, 389-393.

[15] Ries, L.A., Miller, R.W. and Smith, M. (2001) Cancer in Children (Ages 0-14 and Ages 0-19). In: Miller, B., Ries, L.A. and Hankey, B., Eds., SEER Cancer Statistics Review 1973-1990, National Institutes of Health, Bethesda, 21 p.

[16] Magrath, I.T. (1991) African Burkitt's Lymphoma: History, Biology, Clinical Features, and Treatment. The American Journal of Pediatric Hematologyl Oncology, 13, 222-246. https://doi.org/10.1097/00043426-199122000-00019

[17] Magrath, I. (1990) Small Non Cleaved Cell Lymphoma. In: Magrath, I., Ed., The Non-Hodgkin's Lymphomas, Edward Arnold/Hodder and Stoughton, London, 256-278.

[18] Togo, B., Keita, M., Medefo, D., Traore, F. and Sidibe, T. (2008) Le Lymphome de Burkitt à localisation maxillo-faciale en milieu pédiatriqueau CHU Gabriel Touré, Bamako, Mali: A propos de 24 cas. Médecine Tropicale, 68, 600-602.

[19] Adon, A., Vassal, G., Hartmann, O., Couanet, D. and Patte, C. (2008) Les lymphomes malins non hodgkiniens. http://www.igr.fr

[20] Blay, J.-Y., Louis, D., Bouffet, E., Thiesse, P., Biron, P., Favrot, M.C., Brunat-Mentigny, M. and Philip, T. (1991) Management of Non-Hodgkin's Lymphoma. Blood Reviews, 5, 90-97. https://doi.org/10.1016/0268-960X(91)90040-J

[21] Bertrand, Y. and Garnier, N. (2014) Les lymphomes malins non Hodgkiniens de l'enfant et de l'adolescent; Réseau Espace Santé-Cancer Rhône-Alpes; les référentiels, fiche tumeur.

[22] Madanat, F.F., Amr, S.S., Tarawneh, M.S., et al. (1986) Burkitt's Lymphoma in Jordanian Children: Epidemiological and Clinical Study. The Journal of Tropical Medicine and Hygiene, 89, 189-191.

[23] Worch, J., Rohde, M. and Burkhard, B. (2013) Mature B-Cell Lymphoma and Leukemia in Children and Adolescents-Review of Standard Chemotherapy Regimen and Perspectives. Pediatric Hematology and Oncology, 30, 465-483.

https://doi.org/10.3109/08880018.2013.783891 